УДК 658.511:338:3

$10.17213 / 2075-2067-2020-5-182-193$

\title{
ФЕНОМЕН ГАРМОНИЗАЦИИ ПРОИЗВОДСТВА
}

\author{
(C) 2020 г. А. В. Мухин, Г. Э. Ганина, Ю. А. Островский \\ Московский государственный технический университет \\ имени Н. Э. Баумана, г. Москва, Россия
}

Цель статьи. В статье рассматривается новый подход к описанию образа гармоничного производства и методов оценки взаимосвязи гармонии и эффективности на основе построения обобщенных моделей гармонизачии производства.

Результаты исследования. В основе предложенной модели лежит утверждение, гласящее, что «величина производственных затрат эквивалентна вариативности действий при изготовлении продукции». Предлагается использовать универсальный метод производственных затрат для оченки эффективности гармоничного производства через продуцентно-продуктовое соответствие. Любая сфера жизнедеятельности представима в виде взаимосвязи продуцентов и продуктов, в которой элементарной ячейкой жизнедеятельности является элементарное звено «продуцент - продукт», объединяемое геном продуцирования как общим свойством продукта и продуцента. Измерение соответствия между продуктом и продуцентом с помощью гена продуцирования как ёмкости соответствующих свойств одинаковой природь является универсальным методом оценки степени соответствия частей производства.

Перспективы использования результатов. Приведенное в статье описание явления продукиионного ичикла, включающего как действие продуцирования, так и действие репродуцирования, сближает производство, как образеи искусственной системы с образами живой природы, на которые в конечном итоге целесообразно ориентироваться разработчикам при построении гармоничных производственных структур.

Ключевые слова: гармонизация; производственная система; структура производства; функиия производства; продуиентно-продуктовый подход; вариативность; эфрективность.

\section{MANUFACTURING HARMONIZATION PHENOMENA}

(C) 2020 A. V. Muchin, G. E. Ganina, Yu. A. Ostrovskij

\section{Bauman Moscow State Technical University, Moscow, Russia}

Purpose of article. The article considers a new approach to describing the image of harmonious production and methods for assessing the relationship between harmony and efficiency based on the construction of generalized models of production harmonization.

Research result. The proposed model is based on the statement that «the value of production costs is equivalent to the variability of actions in the manufacture of products». It is proposed to use a universal method of production costs to assess the effectiveness of harmonious production through product-product correspondence. Any sphere of life activity is represented as a relationship between producers and products, in which the elementary cell of life activity is an elementary 
link «producer - product», united by the production gene as a common property of the product and the producer. Measuring the correspondence between the product and the producer using the production gene as a capacity of corresponding properties of the same nature is a universal method for assessing the degree of conformity of parts of production.

Prospects for using the results. See description of the phenomenon of a production cycle, which includes both action production and action of reproduction, brings together the production as a sample of the artificial system with images of nature, which in the end, it is advisable to focus developers to build a harmonious industrial structures.

Key words: harmonization; production system; production structure; production function; production-product approach; variability; efficiency.

Введение. В настоящее время наметился новый взгляд на проблему эффективности производственных систем при организации производственной деятельности. Имеющиеся традиционные подходы кеё оценке вызывают озабоченность в связи с тем, что в сферу факторов, определяющих эффективность производства, начинают активно «внедряться» факторы, казалось бы, непроизводственной сферы, а именно: социальные, психологические, поведенческие, морально-этические и т.д. Так как большинство учитываемых факторов по своей природе противоречивы, многие исследователи обращаются к такому понятию, как «гармония производственной системы». Первые шаги было предложено делать в направлении согласования стандартов и описания производственных процессов, образовательных стандартов, например, $[1,2]$.

Принятое определение гармонии в любой системе зиждется на согласованности существования противоречивых факторов, и задачей поиска гармонии является поиск таких факторов и способов установления их значений, которые в совокупности могли бы обеспечить максимальную эффективность системы.

В первую очередь напрашивается и методика решения подобной задачи через построение соответствующих моделей оптимизации. Существующие модели оптимизации охватывают широкий спектр математических методов, включающих как линейные, так и нелинейные модели, динамические и стационарные модели, дискретные и непрерывные зависимости и т.д. В этом смысле нет недостатка в методах проведения оптимизации при организации и управлении машиностроительной производственной системой [3].

Однако в проблеме описания гармонии в производстве возникает существенная трудность. Это происходит всегда, когда в понятие гармонии вкладывается изначальный её смысл, а именно, как только ограничивается количество факторов (противоречивых и противоположных), сразу возникает ощущение неполноты представления о гармонии системы. К сожалению, существующие методики построения моделей оптимизации всегда опираются на ограниченный набор факторов, что объективно приводит к выводу: решенная задача соответствует частному случаю, а не гармонии производства в целом.

В этой связи актуальной проблемой является разработка теоретических положений и практических методик построения обобщенных моделей гармонизации производства на этапах становления, эффективного функционирования и совершенствования производственных процессов. Данный интерес возник на базе сложности, вариативности и противоречивости факторов, определяющих качество производственных процессов.

Специалистов в области экономики и организации производства перестали удовлетворять методы решения производственных задач. Показателен в этом отношении анализ, приведенный в работе Г. Клейнера [4]. Автор убедителен в отношении недостатков существующих методов оценки качества и эффективности производственных процессов. Ни один из существующих подходов, по мнению автора, не позволяет всеобъемлюще оценить процессы производства, хорошо решая лишь частные задачи. 
Автор [4] склоняется к выводу, что на смену частным методам должен придти обобщенный метод, основанный на критерии гармоничности производства и экономики в целом. При этом автор отмечает, что, поставив задачу таким образом, мы оказываемся при этом перед необходимостью найти новый инструментарий поиска решений по критерию гармоничности.

С Г. Клейнером [4] солидарен А.С. Муратов [5], который провел своеобразный обзор работ в области гармонизации экономики производства. Здесь автору удалось заглянуть в историю вопроса о гармонизации экономики. Был охвачен весьма обширный период времени. В число цитируемых авторов вошел, например, Ф. Бастиа [6], работы которого по гармонизации экономики относятся к 1848 г. Не обойден вниманием и один из основоположников теории организации производства К. Адамецки [7], а также А.А. Богданов [8], считающийся одним из основателей всеобщей организационной науки.

Здесь должны быть отмечены и труд Л.М. Семашко [9], посвященный гармоничной цивилизации, и труд А.И. Ивануса [10] по проблеме гармоничного инновационного менеджмента.

Гармонизации производства посвящена монография [11], в которой приведена теория и практика гармонизации производства, в том числе отмечается, что главной проблемой является отсутствие подходящего инструментария для формализации процесса принятия решений по гармонизации. Усугубляет эту проблему то обстоятельство, что, несмотря на давний интерес к гармонизации, не удается даже для ограниченной системы дать полное, исчерпывающее описание ее гармонического состояния. Объясняется это, в первую очередь, тем, что для любой ограниченной системы «толкователь» сталкивается с так называемым «проклятием размерности» (согласно Н. Винеру).

В работе [12] предлагается выполнять экономическую оценку производства, учитывая расширяющуюся информатизацию его инфраструктуры. При внедрении информационных технологий усиливается роль интеллектуальной составляющей производственной системы, соответственно, необходимо учитывать природу критериев оценки.
В предлагаемой классификации разделяются материальные и нематериальные факторы, получившие наименование когнитивных. В число этих факторов включаются объекты, входящие в состав средств технического оснащения производства и информационной поддержки управления производством, объекты организационного обеспечения, производственных знаний, уровень образования сотрудников, способность к анализу, коммуникативные навыки и т.д. Указывается, что перечень может дополняться, что говорит об усложнении модели производственной системы с течением времени.

Действительно, если ограничиться только производством как одной из частей общей системы жизнедеятельности, сразу можно выявить значительное число факторов, которые в реальных условиях часто оказываются противоречивыми [13].

Понятие гармонии является одним из очевидных, когда выступает в качестве интуитивной оценки, и в то же время неуловимо, когда требуется дать четкое, формализованное определение [14], поэтому нужно выявить взаимосвязь между понятиями эффективности и гармоничности производственной системы, так как интуитивно понятно, что они отражают разные категории свойств: эффективность отражает множество «затратных» свойств производства, гармоничность - множество свойств «расположения» производства относительно внешней среды.

Следовательно, для гармонизации производственных процессов в настоящее время необходимо решать две взаимосвязанные задачи:

- создание образа гармоничного производства в виде совокупности элементов, могущих быть объединенными в единое согласованное целое ради достижения замысла производства как системы;

- разработка инструментария формализации отношений между элементами системы и принятия решений по критерию гармоничности.

Методология исследования. Производственная система, созданная человеком, как и любая другая искусственная система, приобретает свойства гармоничности и эффективности в результате целенаправленных действий. Целенаправленность действий 
основана на замысле, определяемом через сравнение с идеалом. Для задачи создания гармоничного производства таким идеалом является гармоничность живой природы. Наблюдая за живой природой, изучая и анализируя, человек многое из нее заимствовал, начиная от источников энергии и заканчивая созданием искусственных технологических устройств как продолжения эффекторов естественной природы.

Что касается гармонии, то здесь дела обстоят гораздо хуже, так как, несмотря на очевидный успех в физике, химии, других естественных науках, человечество находится еще на ранних стадиях изучения механизма возникновения и развития гармонии в живой природе.

Однако целеустремленность человека в этом вопросе позволила достичь некоторых успехов в понимании фрагментов гармонии природы. В первую очередь, здесь речь идет о понятийном аппарате в создании образа гармоничной природы.

Проблеме разработки теоретических положений и практических методик построения обобщенных моделей гармонизации машиностроительного производства посвящены многие исследования, проведенные в МГТУ им. Н.Э. Баумана. В первую очередь следует упомянуть о результатах исследований, приведших к «отчуждению от стоимости» при оценке затрат $[16,17]$. Выразилось это в форме утверждения, гласящего, что «величина производственных затрат эквивалентна вариативности действий при изготовлении продукции». Подобное доказанное утверждение в свою очередь послужило основанием для оценки производственных затрат через соответствие продукта продуценту.

Универсальный метод оценки производственных затрат через продуцентно-продуктовое соответствие оказался плодотворным в смысле всеобщности охвата производственных процессов. Дальнейшие исследования показали, что метод продуцентно-продуктового соответствия применим и для задач построения гармоничного производства.

Этот метод может рассматриваться в качестве инструмента как для задач анализа гармонически действующего производства, так и для задач построения нового производства, соответствующего критерию эффективности.
Предлагается расширение этого метода с использованием элементов, свойственных живой природе, что может быть полезно как для проектировщиков машиностроительных предприятий, так и для широкого круга специалистов промышленного производства, увлеченных идеями гармонизации.

Структура гармоничной живой природы представляет собой огромное по масштабам число элементов, которое, на первый взгляд, очень трудно каким-либо способом упорядочить. Однако специалисты в области биогенеза [15] пришли к выводу, что их можно разделить на два класса: продукты и продуценты.

Активную роль в природе выполняют продуценты (producens - производящий), а термин «продуцирование» закрепился за любым действием, направленным на получение нужного результата. Термин «продуцент» так глубоко проник во многие сферы жизнедеятельности, что им стали обозначать и страны-производители продукции, и системы, производящие товар. В этой связи термину «продуцент» должен соответствовать термин «продукт» как результат продуцирования.

По существу, любую сферу жизнедеятельности можно представить в виде взаимосвязи продуцентов и продуктов. Напрашивается вывод, что элементарной ячейкой жизнедеятельности является элементарное звено «продуцент - продукт», объединяемое геном продуцирования (рис. 1). Свойство продукта, здесь обозначенное как «ген» g, одновременно присуще и продуценту. Таким образом, g выполняет роль связующего

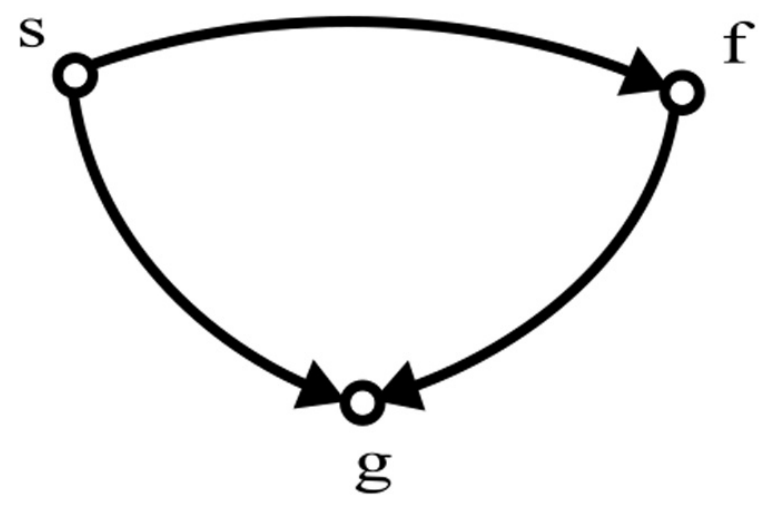

Рис. 1. Элементарная ячейка
продуцирования
s - продуцент; $\mathrm{f}$ - продукт;
$\mathrm{g}$ - ген продуцирования 
звена между s и f, наполняя s и f одинаковым содержанием по виду общих их свойств.

Несмотря на то, что «механизм продуцирования» в живой природе человеком до конца не понят, делается вывод, что он может быть изображен в соответствии с рис. 1. Также делается вывод, что такой механизм может быть использован при построении искусственно созданной системы продуцирования.

Данный прием может быть использован при описании любой искусственно созданной человеком системы жизнедеятельности и, в первую очередь, при описании производства как структуры организованных производственных процессов.

Создать систему, полностью аналогичную системе в живой природе, невозможно по разным причинам, главной из которых является та, что природная система, обладающая функцией продуцирования, имеет еще и функцию репродукции.

Данную функцию имеют все элементы живой природы (для растений, например, пара «дуб — желудь»), но человеку построить её для искусственных систем не представляется возможным. Очевидно, что следует искать пути, имитирующие естественную функцию репродуцирования.

В общем случае, учитывая наличие как продуцирующей, так и репродуцирующей функций, схематично взаимодействие проду-

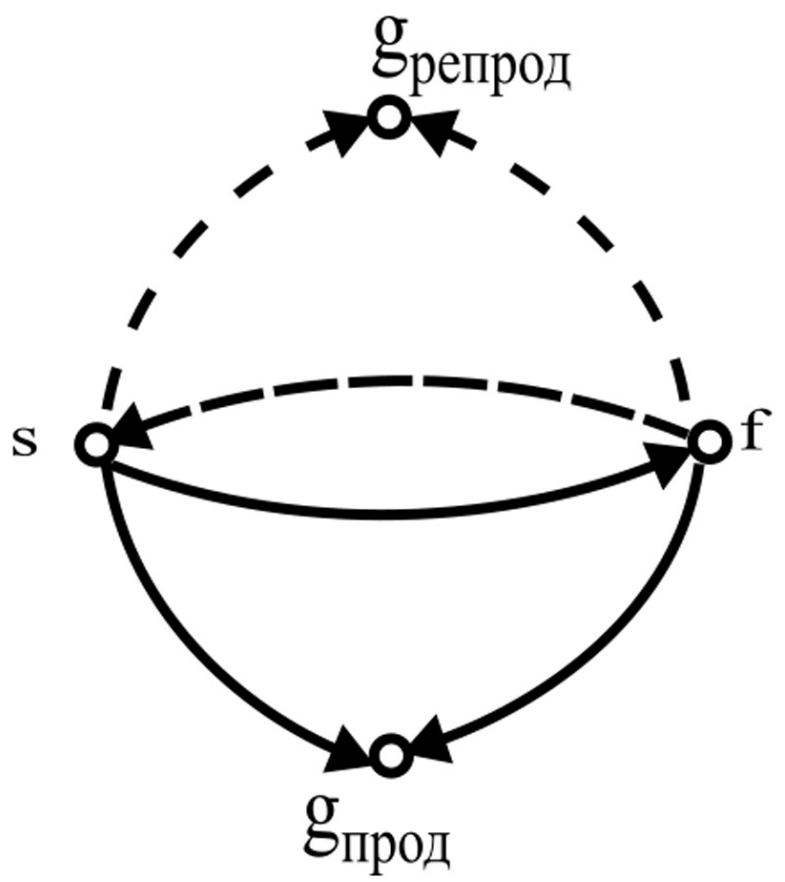

Рис. 2. Схема взаимодействия s и $\mathrm{f}$ центов и продуктов можно изобразить следующим образом (рис. 2).

На рисунке 2: s - продуцент (при продуцировании), продукт (при репродуцировании); f - продукт (при продуцировании), продуцент (при репродуцировании); ( $\rightarrow \mathrm{f}$ ) продуцирование; $(\mathrm{f} \rightarrow \mathrm{s})$ - репродуцирование; $g_{\text {прод }}$ - ген продуцирования; $g_{\text {репрод }}-$ ген репродуцирования.

Как видно из схемы на рис. 2, продукт и продуцент меняются местами в зависимости от функции, которой принадлежат эти структурные элементы.

На основании изложенного можно сделать следующие выводы:

- в качестве образца гармонической системы необходимо брать живую природу во всех её проявлениях;

- в целях упорядочения понятийного аппарата систем жизнедеятельности необходимо ограничиться двумя классами элементов подобных систем: продуктами (f) и продуцентами (s);

- наиболее существенными функциями любой системы жизнедеятельности является продуцирующая (производственная) функция и репродуцирующая функция;

- для создания искусственной системы (в том числе и производственной) можно применять процедуры создания продуцирующих подсистем (подобно созданию многочисленных систем технологического назначения);

- создание подсистем репродуцирования гораздо сложнее в связи с отсутствием подходящих методов формализации репродуцирующих систем в живой природе: для имитации репродуцирующих функций в производственной системе предстоит отыскать новые подходы и методы их создания;

- для практического воплощения идей гармонизации в сфере организации производства необходимо, опираясь на аналоги в живой природе, дать по возможности четкое определение или хотя бы толкование гармоничного производства.

Исходя из сложившегося понятия «гармоничного производства» как системы соразмерных и согласованных частей, соответствующих друг другу [11], и придерживаясь аналогии с живой природой [15], образ гармоничного производства целесообразно строить, опираясь на продуцентно-продукто- 
вый подход. Несмотря на сложность существующих производственных систем, их можно описать одним или множеством звеньев, представленных на рис. 1. При этом, в свою очередь, как s, так и f могут быть материальны (М) или идеальны (J).

Однако такой очевидный подход к описанию гармоничного производства не даёт уверенности в том, что удастся упорядочить и формализовать производственную систему в целом, учитывая следующие обстоятельства:

- как продукты (f), так продуценты (s) могут быть и материальными (M), и идеальными (J);

- необходимость вместе с продуцированием учитывать и функцию репродуцирования говорит о том, что при одних условиях объект является продуцентом, а при других — продуктом;

- любая производственная система представляет собой цепь из связанных звеньев по типу рис. 1, но образ такой цепи не представляется ясным и очевидным.

Каждое из перечисленных обстоятельств должно быть учтено при описании образа гармоничного производства.

Рассмотрим последовательно роль этих обстоятельств в описании образа гармоничного производства.

Специалисты в области технологии и организации производства постоянно имеют дело с многочисленными проявлениями материальной и идеальной формы продуктов и продуцентов. С тем, чтобы свернуть их огромное разнообразие в приемлемую для анализа совокупность, воспользуемся классической предикатной формой.

Все материальные индивиды опишем в форме M(i), где M - свойство «быть материальным». Тогда все материальные продукты в производственной системе будут принадлежать множеству M(f), а продуценты - множеству M(s). К числу M(f) могут быть отнесены все предметы, прошедшие этап изготовления в производстве (детали, узлы, машины и пр.). К числу М(s) могут быть отнесены различные орудия труда (станки, инструменты, оборудование заготовительного производства и др.). Отметим, что перечисленные предметы из числа $\mathrm{M}(\mathrm{f})$ могут быть отнесены к числу $\mathrm{M}(\mathrm{s})$ и наоборот.
Так, любой станок из числа M(s) являлся ранее предметом производства и относился к множеству M(f). «Инверсия», т.е. взаимное превращение продуцента в продукт и наоборот, наталкивает на мысль о возможном существовании репродуцировании в производственной среде.

По аналогии все идеальные индивиды могут быть представлены в виде J(i), где J свойство «быть идеальным». К числу Ј(f) могут быть отнесены идеальные индивиды, являющиеся результатами чьего-либо действия. В качестве примера J(f) может быть названо какоелибо проектное решение по технологическому процессу, результат сравнения с аналогом.

В свою очередь, технологический процесс может быть продуцентом J(s) при выборе технологического оборудования. Здесь также прослеживается «инверсия», т.е. взаимное превращение J(f) и J(s).

В целом на основании сделанных предпосылок можно сформулировать определение производственной системы в терминах продуцентно-продуктовых структур: «если элементы производственной системы являются или продуцентами, или продуктами и выступают или в материальной, или идеальной форме, то производственная система представима в виде множества $\{\mathrm{M}(\mathrm{f}) ; \mathrm{M}(\mathrm{s}) ; \mathrm{J}(\mathrm{f}) ; \mathrm{J}(\mathrm{s})\} »$.

Звено типа (s-f), определяющее какое-либо событие в «жизни» производства, можно создать из упомянутого множества. Важно при этом представить, в какой последовательности расположить созданные звенья.

Основываясь на накопленном опыте по созданию производственных систем, как домашинного, так и машинного, включая компьютеризированное, производства, предложим следующую цепочку событий в жизни производства:

- экспериментальное изготовление материального продукта;

- формализованное описание созданного материального образа продукта;

- формализованное описание последовательности действий по изготовлению материального продукта;

- создание оборудования (продуцента) для изготовления материального продукта.

Используя традиционные термины, можно представить перечисленную цепочку событий следующим образом: 


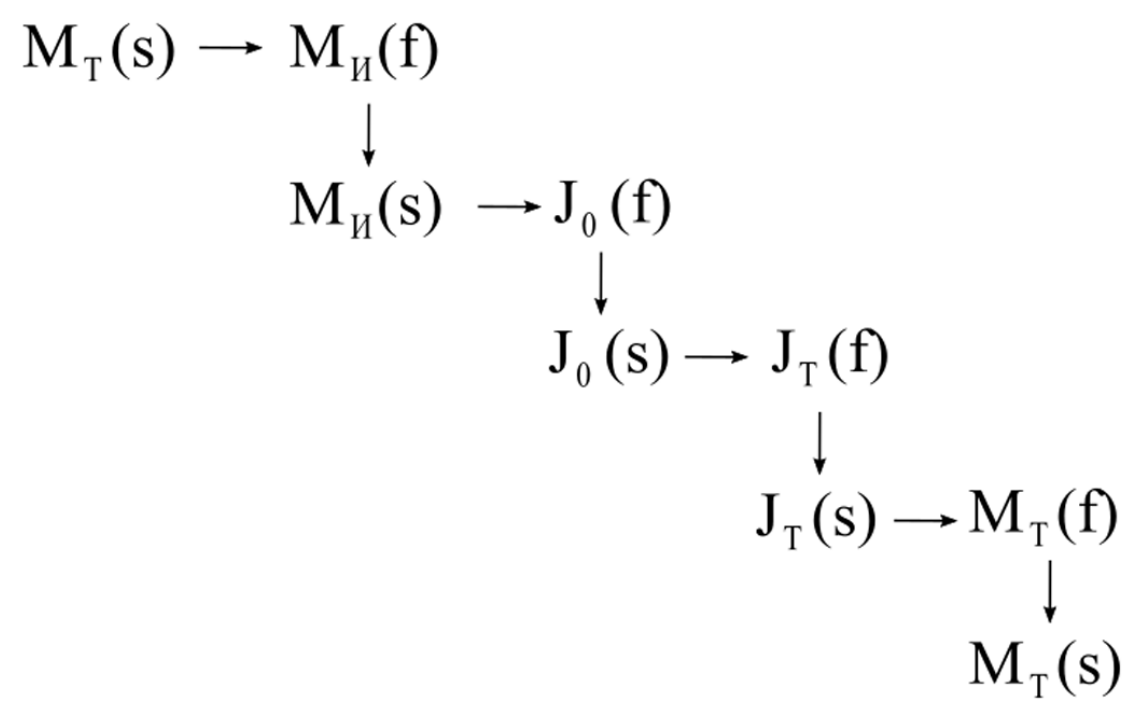

Рис. 3. Каскад последовательности превращений звеньев типа (s-f)

- опытное производство материального продукта (опытно-конструкторская разработка (ОКР));

- проектирование и конструирование материального продукта;

- проектирование технологического процесса (технологическая подготовка производства (ТПП));

- проектирование и изготовление средств технологического оснащения (СТО).

С учетом обозначений элементов производственной системы, возможности их «ин-

$$
\mathrm{M}_{\mathrm{И}}(\mathrm{f}) \sim \mathrm{M}_{\text {И }}(\mathrm{s})
$$

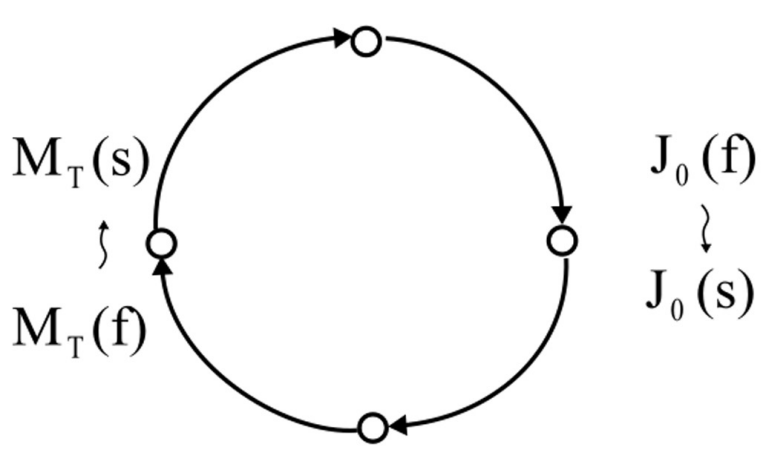

$$
J_{T}(s) \backsim J_{T}(f)
$$

Рис. 4. Продукционный цикл в производстве версии» (из продуцентов в продукты и наоборот), цепочку событий в жизни производства для начала представим в виде каскада (рис. 3).

На рисунке 3: MT(s) - материальный продуцент из числа СТО; МИ(f) - материальный продукт в виде изделия; МИ(s) — материальный продуцент, поставляющий информацию для ОКР; J0(f) - идеальный продукт в виде замыслов и воплощенный в конструкторской документации (КД); J0(s) - идеальный продукт в виде КД, поставляющий информацию для ТПП; JT(f) - идеальный продукт в виде технологических решений; JT(s) - идеальный продуцент в виде технологических решений по созданию СТО; M0(f) - материальный продукт в виде СТО.

Изображенный на рис. 3 каскад можно представить и по-другому (рис. 4). Знаком « $\rightarrow$ на рис. 4 обозначены продуцирующие связи в продуцентно-продуктовом звене, а знаком « >》 обозначен переход продукта в статус продуцента.

Движение по часовой стрелке в цикле отражает функцию продуцирования, а возврат в начало движения отражает функцию репродуцирования.

Продукционный цикл в производстве (рис. 4) состоит из упорядоченной последовательности звеньев $(\mathrm{s} \rightarrow \mathrm{f})$, где каждое звено имеет «собственный» ген продукционности, по которому и можно определить степень гармонизации производства в целом. Рас- 
смотрим гены продукционности по каждому звену и сведем их воедино в таблицу 1.

Степень гармонии (или дисгармонии) в производстве влияет на экономическую эффективность. Можно на примерах показать, как это проявляется для разных звеньев продукционного цикла.

В случае g (MT(s); МИ(f)), как показано в работе [14], при любом отношении ёмкости продуцента от ёмкости продукта возрастают затраты в производстве.

Рассмотрим ситуации, связанные со звеньями 2, 3, 4 (таблица 1). В случае МИ(s) $\rightarrow \mathrm{J0}(\mathrm{f})$ имеет место несогласованность реальных форм и размеров материального продукта (изделия) и совершенство (или несовершенство) языка проектирования в ОКР: возможны ситуации, когда в системе отсутствуют языковые (например, графические) формы, присущие реальному изделию. В автоматизированных системах проектирования это приводит к полной остановке процедуры проектирования, а специалисты в области ОКР в ходе ручной работы (весьма затратной) вынуждены приводить в соответствие реальное изделие и его отображение в КД. В подобной ситуации могут оказаться специалисты, связанные со звеном J0(s) $\rightarrow$ JT(f).

Довольно часто для некоторых решений в ОКР нет готовых ответов в проекте ТПП. Для устранения такого несоответствия при- ходится проводить дополнительные работы, связанные с существенными затратами.

Наконец, для звена JT(s) $\rightarrow$ MT(f) характерна следующая ситуация: спроектированный технологический процесс (через соответствующую JT(s)) требует определенного технологического оборудования (МT(f)), которого ещё нет «в природе» или которое необходимо приобретать или заимствовать в другой продукционной системе. В любом случае, это связано с дополнительными производственными затратами.

В соответствии с правилами гармонизации производства дисгармония наступает, даже если несоответствие между продуцентом или продуктом имеется лишь в одном звене; степень гармоничности производства зависит от числа звеньев, в которых присутствует дисгармония.

Результаты. Приведенные примеры демонстрируют действительную связь показателей гармоничности и эффективности производства; такая связь стала возможной только после того, как на смену калькуляционным методам расчета эффективности пришел структурно-комбинаторный метод, изложенный в [16].

Родился этот метод в связи с тем, что в производстве быстрыми темпами стала нарастать неопределенность экономическо-

\section{Содержание генов продукционности}

Таблица 1

\begin{tabular}{|c|c|c|l|}
\hline № & $\begin{array}{c}\text { Обозначение } \\
\text { звена }\end{array}$ & $\begin{array}{c}\text { Обозначение гена } \\
\text { продукционности }\end{array}$ & \multicolumn{1}{|c|}{ Значение и примеры } \\
\hline 1 & $\mathrm{M}_{\mathrm{T}}(\mathrm{s}) \rightarrow \mathrm{M}_{\mathrm{U}}(\mathrm{f})$ & $\mathrm{g}\left(\mathrm{M}_{\mathrm{T}}(\mathrm{s}) ; \mathrm{M}_{\mathrm{U}}(\mathrm{f})\right)$ & $\begin{array}{l}\text { Отношение «ёмкостей» свойств продукта и дейс- } \\
\text { твий по их достижению: } \\
\mathrm{M}_{\mathrm{U}}(\mathrm{f})=\{\text { форма; размеры }\}, \\
\mathrm{M}_{\mathrm{T}}(\mathrm{s})=\{\text { формообразующие действия }\}\end{array}$ \\
\hline 2 & $\mathrm{M}_{\mathrm{u}}(\mathrm{s}) \rightarrow \mathrm{J}_{0}(\mathrm{f})$ & $\mathrm{g}\left(\mathrm{M}_{\mathrm{U}}(\mathrm{s}) ; \mathrm{J}_{0}(\mathrm{f})\right)$ & $\begin{array}{l}\text { Отношение «ёмкостей» форм и размеров материаль- } \\
\text { ного продукта и символов, их отображающих в КД }\end{array}$ \\
\hline 3 & $\mathrm{~J}_{0}(\mathrm{~s}) \rightarrow \mathrm{J}_{\mathrm{T}}(\mathrm{f})$ & $\mathrm{g}\left(\mathrm{J}_{0}(\mathrm{~s}) ; \mathrm{J}_{\mathrm{T}}(\mathrm{f})\right)$ & $\begin{array}{l}\text { Отношение «ёмкостей» символов изделий и симво- } \\
\text { лов действий по его изготовлению в технологичес- } \\
\text { ком процессе }\end{array}$ \\
\hline 4 & $\mathrm{~J}_{\mathrm{T}}(\mathrm{s}) \rightarrow \mathrm{M}_{\mathrm{T}}(\mathrm{f})$ & $\mathrm{g}\left(\mathrm{J}_{\mathrm{T}}(\mathrm{s}) ; \mathrm{M}_{\mathrm{T}}(\mathrm{f})\right)$ & $\begin{array}{l}\text { Отношение «ёмкостей» символов действий по изго- } \\
\text { товлению материального продукта и технологичес- } \\
\text { кого оборудования, воплощающего эти действия }\end{array}$ \\
\hline
\end{tabular}


го характера, которая в свою очередь, стала следствием такого явления, как ускорение сменяемости изделий в производстве. Явление экономической неопределенности повлекло за собой соответствующие исследования, посвященные разработке новых методов расчета эффективности, появились первые работы [17], посвященные этому направлению. В завершенном виде метод расчета эффективности, отчужденный от стоимости, опубликован в [16].

Оказалось, что этот структурно-комбинаторный метод удобен тем, что с его помощью одинаково успешно можно рассчитывать как эффективность производства на разных этапах жизненного цикла, так и гармоничность.

Особенно это полезно для расчета гармоничности производства, где стоимостные методы оказываются попросту неуместными ввиду специфичности понятия «гармоничность». Применение продукционного подхода здесь позволяет применять совместно показатели, содержащие свойства из разных категорий. Вначале «феномен гармоничности» работал на формирование структуры производства в виде «продуцентно-продуктового» отношения, а затем новый структурно-комбинаторный метод расчета эффективности оказался пригоден для оценки гармоничности производства.

Заключение. Рассмотренная в статье методология гармонизации производства опирается на ряд положений, среди которых можно выделить главные:

- продуцентно-продуктовый подход очевиден в том смысле, что для производства единственно подходящим аналогом в плане гармонизации является живая природа «прародительница» термина «продуцент» и таких взаимосвязанных явлений, как продуцирование и репродуцирование;

- универсальным методом оценки степени соответствия частей производственного процесса является измерение соответствия между продуктом и продуцентом с помощью гена продуцирования как ёмкости соответствующих свойств одинаковой природы;

- введенное понятие «продукционный цикл» позволило для множества производственных ситуаций объединить в единое целое связанные действия продуцирования и репродуцирования.
Построенная на основе главных положений методология гармонизации производственных процессов носит концептуальный характер и позволяет развивать и решать многочисленные задачи организации производства на единой онтологической базе.

\section{Литература}

1. Electrical Codes, Standards, Recommended Practices and Regulations, Editor (s): Robert J. Alonzo, William Andrew Publishing, 2010.

2. Quintino L., Fernandes I., Miranda R.M. International harmonization of training and qualification in the manufacturing industry. Journal of European Industrial Training. 2011. 35. 502514. DOI:10.1108/03090591111138044.

3. Косачев А. В., Лялин В. Е., Семенов В.В. Модель оптимального управления долгосрочным развитием интеллектуального предприятия // Аудит и финансовый анализ. 2006. 一 №4. - С. 314-349.

4. Клейнер Г. Экономика должна быть гармоничной // Современная конкуренция. 2009. - №2 (14). - C. 19-21.

5. Муратов А. С. Гармонизационный подход к экономике и управлению предприятиями: теория и практика: монография. - М.: Креативная экономика, 2011.

6. Бастиа Ф. Экономические гармонии. - М.: ЭКСМО, 2007.

7. Адамецєки K. О науке организации. М.: Экономика, 1972.

8. Богданов А. А. Тектология: всеобщая организационная наука. - М.: Финансы, 2003.

9. Гармоничная цивилизация. Инновационные проекты ГСГ / Л.М. Семашко. Санкт-Петербург: Лита, 2009.

10. Иванус А.И. Гармоничный инновационный менеджмент. - M.: URSS, 2011. $248 \mathrm{c.}$

11. Клевлин А.И. Логистические основы организации крупных промышленных комплексов. - Тольятти-Самара: ИЧП «Акцент», 1996.

12. Omelchenko I., Drogovoz P., Gorlacheva E., ShiboldenkovV., Yusufova O. The modeling of the efficiency in the new generation manufacturing-distributive systems based on the cognitive production factors. // IOP Conf. Series: 
Materials Science and Engineering 630 (2019) 012020, IOP Publishing, DOI:10.1088/1757899X/630/1/012020.

13. Силинский В. С. Гармонизация сбытовой и производственной деятельности высокотехнологичного промышленного предприятия в условиях неопределенности и нестабильности // Экономический журнал. 2015. - №1 (37). - C. 6-17.

14. Самохвалова В.И. О метафизическом смысле гармонии. // Дельфис. - 1998. №16 (3/1998).

15. Цавкелова Е.А., Климова С.Ю., Чедынцева Т.А., Нетрусов А.И. Микроорганизмы - продуценты стимуляторов роста растений и их практическое применение (обзор) // Прикладная биохимия и микробиология. №2 (42). - 2006. - С. 133-143.

16. Мухин А.В., Ганина Г.Э., Островский Ю.А. Учет эффективности производства в систем технико-экономических показателей промышленного предприятия // Контроллинг. - 2014. - №52. - С. 26-35.

17. Мухин А.В., Островский Ю.А., Петров B. B. Метод оптимального распределения ресурсов при решении производственных задач в условиях экономической неопределенности // СТИН. - 2013. - №2. C. $35-40$.

\section{References}

1. Electrical Codes, Standards, Recommended Practices and Regulations, Editor (s): Robert J. Alonzo, William Andrew Publishing, 2010.

2. Quintino L., Fernandes I., Miranda R.M. International harmonization of training and qualification in the manufacturing industry. Journal of European Industrial Training. 2011. 35. 502514. DOI:10.1108/03090591111138044.

3. Kosachev A. V., Ljalin V.E., Semenov V.V. Model' optimal'nogo upravlenija dolgosrochnym razvitiem intellektual'nogo predprijatija [Model of optimal management of long-term development of an intellectual enterprise].// Audit i finansovyj analiz [Audit and financial analysis]. - 2006. — №4. — Pp. 314-349.

4. Klejner $G$. Jekonomika dolzhna byt' garmonichnoj [The economy should be harmonious]// Sovremennaja konkurencija [Modern competition]. — 2009. — №2 (14). — Pp. 19-21.
5. Muratov A.S. Garmonizacionnyj podhod $\mathrm{k}$ jekonomike i upravleniju predprijatijami: teorija i praktika: monografija [Harmonization approach to the economy and management of enterprises: theory and practice: monograph]. Moscow: Kreativnaja jekonomika, 2011.

6. Bastia F. Jekonomicheskie garmonii [Economic harmony]. - Moscow: JeKSMO, 2007.

7. Adamecki $K$. O nauke organizacii [On the science of organization]. - Moscow: Jekonomika, 1972.

8. Bogdanov A.A. Tektologija: vseobshhaja organizacionnaja nauka [Tectology: General organizational science]. - Moscow: Finansy, 2003.

9. Garmonichnaja civilizacija. Innovacionnye proekty GSG [Harmonious civilization. Innovative projects of GSG] / L. M. Semashko. Saint-Petersburg: Lita, 2009.

10. Ivanus A.I. Garmonichnyj innovacionnyj menedzhment [Harmonious innovation management]. — Moscow: URSS, 2011. — 248 p.

11. Klevlin A.I. Logisticheskie osnovy organizacii krupnyh promyshlennyh kompleksov [Logistics bases of organization of large industrial complexes]. — Tol'jatti-Samara: IChP «Akcent», 1996.

12. Omelchenko I., Drogovoz P., Gorlacheva E., Shiboldenkov V., Yusufova O. The modeling of the efficiency in the new generation manufacturing-distributive systems based on the cognitive production factors. // IOP Conf. Series: Materials Science and Engineering 630 (2019) 012020, IOP Publishing, DOI:10.1088/1757899X/630/1/012020.

13. Silinskij V.S. Garmonizacija sbytovoj i proizvodstvennoj dejatel'nosti vysokotehnologichnogo promyshlennogo predprijatija v uslovijah neopredelennosti i nestabil'nosti [Harmonization of sales and production activities of a high-tech industrial enterprise in conditions of uncertainty and instability] // Jekonomicheskij zhurnal [Economic journal]. - 2015. №1 (37). - Pp. 6-17.

14. Samohvalova V.I. O metafizicheskom smysle garmonii [On the metaphysical meaning of harmony] // Del'fis. - 1998. - №16(3/1998).

15. Cavkelova E. A., Klimova S. Ju., Chedynceva T.A., Netrusov A.I. Mikroorganizmy producenty stimuljatorov rosta rastenij $\mathrm{i}$ ih prakticheskoe primenenie (obzor) [Microorganismsproducers of plant growth stimulators and their 
practical application (review)] // Prikladnaja biohimija i mikrobiologija [Applied biochemistry and Microbiology]. — №2 (42). — 2006. - Pp. 133-143.

16. Muhin A.V., Ganina G. Je., OstrovskijJu.A. Uchet jeffektivnosti proizvodstva $\mathrm{v}$ sistem tehniko-jekonomicheskih pokazatelej promyshlennogo predprijatija [Accounting for production efficiency in the system of technical and economic indicators of an industrial enter- prise] // Kontrolling [Controlling]. — 2014. №52. - Pp. 26-35.

17. Muhin A. V., Ostrovskij Ju. A., Petrov V.V. Metod optimal'nogo raspredelenija resursov pri reshenii proizvodstvennyh zadach $\mathrm{v}$ uslovijah jekonomicheskoj neopredelennosti [Method of optimal resource allocation for solving production problems in conditions of economic uncertainty] // STIN. - 2013. - №2. - Pp. 35-40.

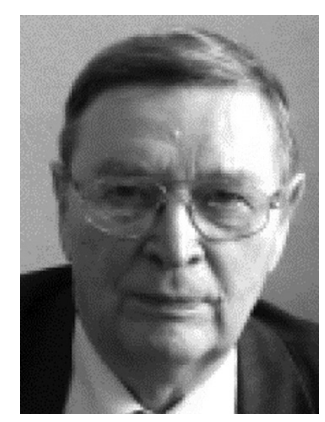

105005, г. Москва, ул. 2-я Бауманская, 7

7 2nd Baumanskaya st., 105005, Moscow, Russia

E-mail: alvasmuhin@yandex.ru 


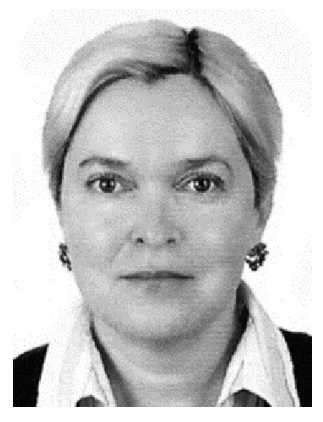

Ганина Галина Эдуардовна - кандидат технических наук, доцент кафедры «Экономика и организация производства» Московского государственного технического университета им. Н.Э. Баумана, специалист в области организации производства и инновационного менеджмента, автор более 40 научных статей и 2 учебных пособий.

Ganina Galina Eduardovna - Candidate of Engineering Sciences, Associate Professor, Department of Economics and Production Organization, Bauman Moscow State Technical University, specialist in the field of production organization and innovation management, author of more than 40 scientific articles and 2 textbooks.

105005 , г. Москва, ул. 2-я Бауманская, 7

7 2nd Baumanskaya st., 105005, Moscow, Russia

E-mail: galya.ganina@yandex.ru

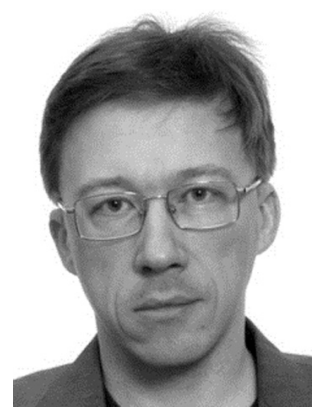

Островский Юрий Андреевич - кандидат технических наук, доцент кафедры «Метрология» Московского государственного технического университета им. Н.Э. Баумана, специалист в области автоматизации технологической подготовки производства, автор более 30 научных статей.

Ostrovsky Yuri Andreevich - Candidate of Engineering Sciences, Associate Professor, Department of Metrology, Bauman Moscow State Technical University, specialist in the field of automation of technological preparation of production, author of more than 30 scientific articles.

105005 , г. Москва, ул. 2-я Бауманская, 5

5 2nd Baumanskaya st., 105005, Moscow, Russia

E-mail: yost@bmstu.ru 